\title{
Le protocole de Kyoto, le clivage Nord-Sud et le challenge du développement durable
}

\author{
Moïse Tsayem Demaze \\ Maître de Conférences \\ Université du Maine, UMR CNRS 6590 ESO, équipe GREGUM \\ Chercheur associé à l'IRD, US 140 ESPACE \\ Moise.Tsayem_Demaze@univ-lemans.fr \\ Avenue Olivier Messiaen \\ 72085 Le Mans Cedex 9
}

\begin{abstract}
Résumé
Pour lutter contre le réchauffement de la Terre, le protocole de Kyoto a prescrit pour 2008-2012, par rapport à 1990, une réduction d'au moins 5\% des émissions mondiales de gaz à effet de serre d'origine anthropique. La réduction est exigée aux pays développés, une dispense étant accordée aux pays en développement et aux pays émergents. Le clivage ainsi entériné signifie que les pays développés doivent se préoccuper du changement climatique pendant que les autres pays se préoccupent de leur développement. Ce clivage est analysé dans cet article à l'aide des données économiques et environnementales les plus récentes. Le croisement de l'Indice de Développement Humain (IDH) et de l'Empreinte Ecologique (E.E.) permet d'aborder la double exigence du développement durable pour chaque pays : avoir une E.E. inférieure ou égale à $2,1 \mathrm{hg} / \mathrm{habitant}$ et un IDH supérieur ou égal à 0,800 . Seuls Cuba et Trinidad et Tobago semblent satisfaire à cette double exigence qui constitue un défi majeur que la mise en œuvre du développement durable pose au monde : concilier protection de l'environnement et développement socio-économique en impliquant les pays du Nord et ceux du Sud.
\end{abstract}

Mots-clés. Emissions de gaz à effet de serre, empreinte écologique, indicateur de développement humain, développement durable, protocole de Kyoto.

\begin{abstract}
Kyoto protocol, North-South cleavage and sustainable development challenge To fight against global warming, the Kyoto protocol prescribed for 2008-2012, compared to 1990, a reduction from at least $5 \%$ of the world greenhouse gas emissions generated by human activities. The reduction is requested from developed countries, an exemption being granted to developing and emerging countries. The cleavage thus ratified means that developed countries must be involved in the climate change struggle while other countries are involved in their development. This cleavage is analyzed in this paper with the most recent economic and environmental data. By crossing the Index of Human development (IDH) and the Ecological Footprint (E.E.), we address the double requirement of sustainable development for each country: having an E.E equal or lower than $2,1 \mathrm{hg} / \mathrm{inh}$ abitant with an IDH equal or higher than 0,800 . Only Cuba and Trinidad and Tobago seem to satisfy this double requirement which constitutes a major challenge that the implementation of sustainable development poses at the world: protecting environment together with socio-economic development by implying developed and developing countries.
\end{abstract}

Key words. Greenhouse gas emissions, ecological footprint, human development index, sustainable development, protocol of Kyoto. 


\section{Introduction}

Le protocole de Kyoto a été élaboré en 1997 pour servir de cadre international à la lutte contre le réchauffement de la Terre. Il complète la convention des Nations unies sur le changement climatique qui a été adoptée en 1992 au sommet de Rio de Janeiro consacré aux problèmes d'environnement et de développement dans le monde. L'objectif de ce protocole est de parvenir à la limitation ou à la réduction des émissions mondiales de gaz à effet de serre d'origine anthropique durant la période 2008-2012, de sorte qu'au terme de cette période, les émissions ne soient pas supérieures à celles de 1990. Pour que cet objectif soit atteint, le protocole a préconisé une baisse globale d'au moins $5 \%$, en définissant et en répartissant des quotas d'émissions entre les pays ou les groupes de pays qui ratifient le protocole (Tsayem, 2008a).

L'essentiel de l'effort de réduction repose sur les pays développés et les pays d'Europe de l'Est à économie en transition. Les pays en développement, y compris les pays dits émergents comme la Chine ou le Brésil, sont dispensés d'engagements contraignants de réduction. Cette différence de traitement a conduit à une sorte de bipolarisation, avec d'un côté les pays développés, ou pays du Nord, appelés à fournir les efforts nécessaires, et de l'autre côté les pays en développement, ou pays du Sud, appelés à accorder la priorité à la lutte contre la pauvreté pour sortir du sous-développement (Riedacker, 2003 ; Quesnault, 2005 et 2006).

En dispensant les pays en développement et les pays émergents, le protocole de Kyoto considère que ces pays doivent se développer, en augmentant au besoin leurs émissions de gaz à effet de serre et donc en dégradant l'environnement comme les pays aujourd'hui développés qui sont accusés de s'être développés en dégradant le climat et l'environnement. Le protocole admet ainsi la dichotomie entre développement et environnement et considère même que le développement est synonyme de dégradation de l'environnement. Qu'en est-il alors du développement durable? N'est-il pas censé concilier l'environnement et le développement? Comment parvenir à ce développement durable en impliquant les pays développés et les pays en développement?

Cet article apporte des éléments de réponse à ces interrogations à partir du traitement et de l'analyse des données économiques et environnementales les plus récentes. Les statistiques sur les émissions de gaz à effet de serre par pays entre 1990 et 2005 permettent de faire le point sur les efforts de réduction à fournir entre 2008 et 2012 en vue de juguler le réchauffement de la Terre qui est maintenant communément admis. Les représentations cartographiques et graphiques offrent une esquisse géopolitique qui situe les pays du Nord et ceux du Sud par rapport aux enjeux de développement et de protection de l'environnement. Le développement durable est abordé au regard du croisement de l'Empreinte Ecologique (EE) et de l'Indice de Développement Humain (IDH).

L'objectif de l'article n'est pas de faire une critique des liens conceptuels entre pays du Nord et pays du Sud ou entre développement et environnement, ou encore entre développement et développement durable. Il ne s'agit pas non plus de faire une revue de la littérature géographique sur le développement durable ou une critique des indicateurs économiques et écologiques qui sous-tendent le développement durable ${ }^{1}$. Il s'agit essentiellement d'utiliser ces indicateurs dans une optique pédagogique pour aborder le développement, l'environnement et le développement durable dans une perspective géopolitique montrant la difficulté et la complexité de la mise en œuvre du développement durable tant pour les pays développés que pour les pays en développement.

\section{Le clivage Nord-Sud redessiné par la convention climat et par le protocole de Kyoto}

L'adoption du protocole de Kyoto en 1997 a représenté l'aboutissement de négociations politiques opposant principalement les Etats-Unis, l'Union Européenne et les pays en développement. Ce protocole a entériné une bipolarisation déjà esquissée dans la convention sur le changement climatique

\footnotetext{
${ }^{1}$ Pour un aperçu de la littérature géographique française sur le développement durable, voir, entre autres, L'Information Géographique, n³, 2007 ; Mancebo, 2006 ; Veyret et Arnould, 2008 ; Arnould et Simon, 2007 ; Veyret (dir.), 2007 ; Da Lage et al., 2008; Miossec et al., 2004; Carroué (dir.), 2006 ; Froger, 2006. Pour une critique des indicateurs du développement durable, voir notamment Arnould et Vincent, 2004 ; Boisvert, 2005 ; Theys, 2001.
} 
qui a défini un groupe de pays dits de l'Annexe I. Ce sont des pays développés associés aux pays d'Europe de l'Est dont les économies sont dites en transition ${ }^{2}$. Ce premier pôle constitue en d'autres termes ce qu'on appelle pays du Nord (fig. 1). Le deuxième pôle rassemble le reste du monde, c'est-àdire les pays en développement, y compris les pays émergents. Ce deuxième pôle constitue ce qu'on appelle pays du Sud.

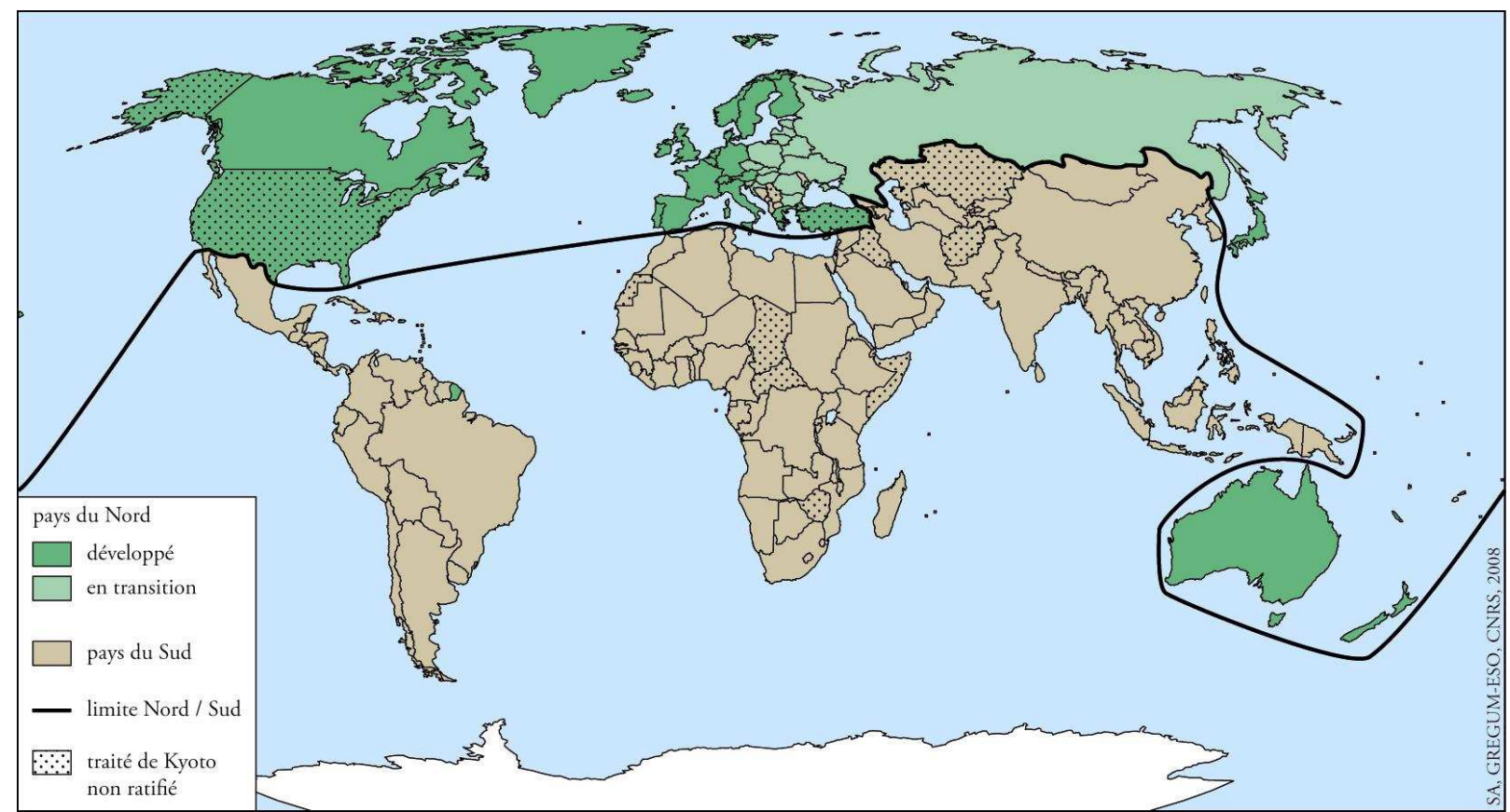

Figure 1. Le clivage Nord-Sud d'après la convention climat et le protocole de Kyoto

Ce découpage Nord-Sud est semblable à quelques exceptions près aux autres découpages géopolitiques distinguant le Nord et le Sud (Capedepuy, 2007). On remarque que le Nord comporte des pays situés au Sud de l'équateur (Australie et Nouvelle-Zélande), que le Sud englobe des pays pauvres et des pays émergents comme la Chine, le Brésil, l'Inde ou l'Argentine, que des pays d'Europe de l'Est (Moldavie, Serbie,...) appartiennent au Sud alors qu'ils sont situés au Nord de l'équateur. D'après Brandt, d'une manière générale et bien qu'il n'y ait pas de classification uniforme ou permanente, "Nord " et "Sud " sont synonymes grosso modo de "riche » et de "pauvre ", de pays « développés » et de pays « en voie de développement » (cité par Capedepuy, 2007).

La communauté internationale a adopté ce clivage Nord-Sud à Rio de Janeiro et à Kyoto en le considérant comme principe de base pour la répartition des efforts à fournir dans le cadre de la lutte contre le changement climatique, étant entendu que les engagements et les efforts demandés aux pays $\mathrm{du}$ Nord sont distincts de ceux demandés aux pays du Sud. Ainsi, la convention sur le changement climatique indique dans son article 3 que sur la base de "l'équité » et des « responsabilités communes mais différenciées ", les pays développés doivent être à l'avant-garde de la lutte contre les changements climatiques (principe d'équité et de responsabilités communes mais différenciées). Cet article ajoute que les «besoins spécifiques» et la "situation spéciale » des pays en développement doivent être pris en compte dans la répartition des efforts à fournir en vue de résorber la crise climatique et ses conséquences (principe de la faible implication des pays en développement). L'alinéa 2 de l'article 4 précise que les pays développés ainsi que l'ensemble des pays figurant dans l'Annexe I doivent prendre des engagements spécifiques contenant des mesures et politiques visant à réduire leurs

\footnotetext{
${ }^{2}$ Allemagne, Australie, Autriche, Belarus, Belgique, Bulgarie, Canada, Croatie, Danemark, Espagne, Estonie, Etats-Unis, Fédération de Russie, Finlande, France, Grèce, Hongrie, Irlande, Islande, Italie, Japon, Latvia, Liechtenstein, Lituanie, Luxembourg, Monaco, Nouvelle Zélande, Norvège, Pays Bas, Pologne, Portugal, République Tchèque, Royaume Uni, Slovénie, Slovaquie, Suède, Suisse, Turquie, Ukraine, Union européenne.
} 
émissions de gaz à effet de serre pour les ramener à leur niveau de 1990. Les pays développés doivent en outre fournir des « ressources financières nouvelles et additionnelles pour couvrir la totalité des coûts convenus encourus par les pays en développement du fait de l'exécution de leurs obligations conformément à la convention (art. 4, alinéa 3). En d'autres termes, l'application de la convention par les pays en développement est dépendante des crédits alloués à cet effet par les pays riches qui sont tenus de supporter les coûts financiers de la lutte contre le réchauffement climatique en raison du fait que le développement de ces pays est accusé d'avoir généré la crise climatique actuelle. C'est le principe de la «responsabilité historique » des pays développés ${ }^{3}$.

Ce principe qui a fait l'objet de consens politique met explicitement en cause les pays développés, admettant que leur développement est à l'origine de la dégradation du climat et de l'environnement mondial. Les pays en développement et les pays émergents (Chine, Mexique, Brésil, ...) sont exemptés de réduction contraignante, leur développement économique et social étant censé entraîner inéluctablement une augmentation conséquente de leurs émissions de gaz à effet de serre ainsi qu'il en a été dans les pays du Nord. Les pays en développement sont ainsi jugés non ou peu responsables du réchauffement actuel. La convention et le protocole reconnaissent que ces pays en développement doivent s'occuper prioritairement de leur croissance économique et de la lutte contre la pauvreté. Or, en exonérant les pays en développement et les pays émergents, le principe de la responsabilité historique des pays développés admet que les pays aujourd'hui non développés doivent se développer à leur tour même si cela doit aussi dégrader le climat et l'environnement. Cette posture suppose que le développement de ces pays se réalise de la même manière que celui des pays déjà développés, ce qui sous-entend que la crise climatique perdurera parallèlement au développement des pays.

L'inscription de ce principe dans la convention et dans le protocole de Kyoto a constitué un acte géopolitique majeur dont les implications semblent avoir été sous-estimées. C'est sur la base de ce principe que les pays non développés refusent de prendre des engagements chiffrés de réduction de leurs émissions de gaz à effet de serre dans le cadre des négociations en cours pour l'après Kyoto (Alternatives Sud, 2006). Ainsi se pose la question de la gouvernance et de la coopération Nord-Sud, ainsi que celle de la solidarité internationale en matière de protection de l'environnement planétaire et de promotion du développement durable (Quenault, 2006 ; Tsayem, 2008a et b). Des pays émergents comme la Chine, l'Inde ou l'Afrique du Sud, envisagent de réduire leurs émissions au mieux après 2020, sinon à l'horizon $2050^{4}$. Cette situation amène à s'interroger sur l'efficacité de la lutte contre le changement climatique à court, moyen et long terme. Quel impact climatique aura la réduction des émissions des pays du Nord si les pays du Sud accroissent leurs émissions ? Cette interrogation est d'autant plus préoccupante que les réductions demandées aux pays développés ne représentent globalement que 5\% de réduction totale à l'horizon 2012:8\% de réduction pour les pays de l'Union européenne (comprenant alors 15 pays), $7 \%$ de réduction pour les Etats-Unis, $6 \%$ de réduction pour le Japon ainsi que pour le Canada, statu quo pour la Russie et pour l'Ukraine, etc.

Le protocole de Kyoto est entré en vigueur en février 2005 après sa ratification par la Russie à la fin de l'année 2004. L'Australie a ratifié le protocole en décembre 2007, ce qui porte à 175 le nombre de pays qui ont adhéré au protocole et sont donc tenus de l'appliquer entre 2008 et 2012. Les Etats-Unis sont le seul grand pays développé qui n'a pas ratifié ce protocole, comme la Turquie (qui fait partie des pays de l'Annexe I) ou comme quelques pays du Sud (fig. 1). Pourtant le réchauffement de la Terre ne fait plus l'objet de contestation.

\section{Accroissement de l'effet de serre et réchauffement de la Terre}

Le réchauffement actuel de la Terre est aujourd'hui admis de manière quasi-unanime alors qu'il a fait l'objet de contestations et de polémiques au cours de cette décennie (Leroux, 2002). D'après le Groupe Intergouvernemental d'Experts sur l'Evolution du Climat (GIEC), qui est en quelque sorte le porte parole mondiale de l'explication de ce réchauffement de la Terre, ce réchauffement est causé par

\footnotetext{
${ }^{3}$ en quelque sorte le principe pollueur payeur, exigeant que les pays développés payent ou réparent les dégradations environnementales que leur développement a engendrées.

${ }^{4}$ Voir le dossier consacré à ce sujet dans Alternatives Internationales, $n^{\circ} 41$, décembre 2008.
} 
l'augmentation des concentrations atmosphériques mondiales de gaz à effet de serre depuis l'ère industrielle (tabl. 1)

\begin{tabular}{|l|l|l|l|}
\cline { 2 - 4 } \multicolumn{1}{c|}{} & $\mathbf{C O}_{\mathbf{2}}$ & $\mathbf{C H}_{\mathbf{4}}$ & $\mathbf{N}_{\mathbf{2}} \mathbf{0}$ \\
\hline Avant la révolution industrielle (1750) & $280 \mathrm{ppm}$ & $715 \mathrm{ppb}$ & $270 \mathrm{ppb}$ \\
\hline En 2005 & $379 \mathrm{ppm}$ & $1774 \mathrm{ppb}$ & $319 \mathrm{ppb}$ \\
\hline
\end{tabular}

Tableau 1. Evolution des concentrations atmosphériques mondiales pour quelques gaz à effet de serre (source: GIEC, 2007).

$\mathrm{CO}_{2}$ : dioxyde de carbone ; $\mathrm{CH}_{4}$ : méthane ; $\mathrm{N}_{2} \mathrm{O}$ : protoxyde d'azote. ppm: partie par million; ppb: partie par billion.

Cette augmentation des concentrations atmosphériques des gaz à effet de serre est attribuée à l'accroissement continu des émissions de gaz issus d'activités économiques, agricoles et industrielles. Il s'agit donc d'émissions essentiellement d'origine anthropique qui renforcent l'effet de serre. C'est surtout l'augmentation des émissions de $\mathrm{CO}_{2}$ qui est mise en cause ; elle contribue pour $52 \%$ à l'augmentation de l'effet de serre (GIEC, 2001). L'augmentation de l'effet de serre est considérée comme la cause principale du réchauffement climatique actuel car elle entraîne une hausse des températures moyennes à la surface de la Terre ${ }^{5}$. Les données de l'Organisation Météorologique Mondiale (OMM) montrent que les années les plus chaudes sont les plus récentes ${ }^{6}$. Le GIEC (2007) indique que «l'essentiel de l'accroissement observé sur la température moyenne globale depuis le milieu du 20ème siècle est très vraisemblablement dû à l'augmentation observée des gaz à effet de serre anthropiques ». Les facteurs humains du réchauffement, qui interfèrent avec les facteurs naturels, ne sont plus vigoureusement discutés. Plusieurs activités anthropiques sont mises en cause: combustion du charbon, du gaz naturel et du pétrole, industries, transports, urbanisation, élevage, déforestation, etc.

Le dernier rapport du GIEC (2007) fait état d'une augmentation de la température moyenne de la Terre de l'ordre de $0,74^{\circ} \mathrm{C}$ durant le $20^{\text {ème }}$ siècle (d'après les enregistrements effectués dans les stations météorologiques entre 1906 et 2005). Les simulations suggèrent une hausse supplémentaire de $0,6^{\circ} \mathrm{C}$ à la fin du $21^{\text {ème }}$ siècle (2090-2099) dans l'hypothèse du maintien du niveau de concentration atmosphérique des gaz à effet de serre de l'année 2000. D'autres scénarios indiquent une hausse entre $1,8^{\circ} \mathrm{C}$ et $4^{\circ} \mathrm{C}$, avec un intervalle de confiance qui varie entre $1,1^{\circ} \mathrm{C}$ et $6,4^{\circ} \mathrm{C}$ (GIEC, 2007).

\section{Evolution des émissions mondiales de gaz à effet de serre}

Les statistiques relatives aux émissions de gaz à effet de serre dans le monde sont disparates et peu homogènes. Des efforts importants sont fournis depuis quelques années par les organisations internationales (Banque Mondiale, Nations unies, Union européenne, FAO, PNUE) pour collecter et diffuser ces statistiques. Les données sur le $\mathrm{CO}_{2}$ sont les plus diffusées. Les pays qui ont ratifié le protocole de Kyoto, et en particulier les pays de l'Annexe I, sont tenus de transmettre annuellement leurs inventaires au secrétariat de la convention sur le changement climatique qui compile et diffuse les données (http://unfccc.int/ghg_data/).

Les émissions mondiales de gaz à effet de serre étaient de 33 milliards 144 millions de tonnes d'équivalent $\mathrm{CO}_{2}$ en 1990 (tabl. 2). Ces données montrent la prédominance des pays du Nord dans les émissions, avec celles des Etats-Unis qui représentaient 19\% des émissions totales. Avec 13\%, les émissions des pays asiatiques étaient du même ordre que celles des pays de l'Union européenne.

Une étude récente confirme pour l'année 2000 la position des pays du Nord en tant que plus grands émetteurs de gaz à effet de serre (tabl. 3). Les données de 2000, comparées à celles de 1990, indiquent

\footnotetext{
${ }^{5}$ Ceux qui réfutent le réchauffement de la Terre, comme Leroux (2002), considèrent que c'est l'augmentation des températures qui expliquent l'augmentation de l'effet de serre. Ces auteurs préfèrent l'expression changement climatique à réchauffement de la Terre ou réchauffement climatique (Allègre, 2007).

${ }^{6} 11$ des 12 dernières années sont les plus chaudes depuis que les mesures sont effectuées.
} 
une augmentation des émissions aussi bien pour les pays du Nord, Etats-Unis en tête, que pour les pays en développement et les pays émergents comme la Chine dont les émissions totales représentent $14 \%$ des émissions mondiales même si par habitant ces émissions chinoises sont de l'ordre de 1\% (tabl. 3). La répartition entre pays développés et pays en développement est presque égale pour les émissions totales alors que la différence d'émissions par habitant est quatre fois plus élevée dans les pays développés que dans les pays en développement. La « responsabilité historique » des pays développés est caractérisée par le fait que ces pays ont émis $77 \%$ du $\mathrm{CO}_{2}$ d'origine fossile durant la période 18502000 , contre $23 \%$ pour les pays en développement.

\begin{tabular}{llll}
\hline & $\begin{array}{l}\text { total } \\
\left(\mathrm{Mt} \text { éq } \mathrm{CO}_{2}\right)\end{array}$ & tonnes/hab & $\%$ du total \\
\hline États-Unis & 6231 & 24,9 & 19 \\
\hline Pays TEM & 6032 & 14,6 & 18 \\
\hline Russie & 4706 & 16,3 & 14 \\
\hline Union europ. (les 15) & 4275 & 11 & 13 \\
\hline Chine, Corée, Asie S.E. & 4176 & 3,3 & 13 \\
\hline Amérique latine & 2850 & 6,5 & 9 \\
\hline Sous-continent indien & 2254 & 1,9 & 7 \\
\hline Afrique & 2188 & 3,4 & 7 \\
\hline Europe de l'Est & 1326 & 10,7 & 4 \\
\hline Japon & 1259 & 10,2 & 4 \\
\hline Moyen-Orient & 1160 & 5,6 & 3 \\
\hline Canada & 597 & 22,1 & 2 \\
\hline Océanie & 464 & 20,2 & 1 \\
\hline Total Monde & 33144 & 6,3 & 100 \\
\hline
\end{tabular}

MtéqCO $\mathrm{CO}_{2}$ : millions de tonne d'équivalent $\mathrm{CO}_{2} ; \mathrm{TEM}$ : pays de l'ex bloc soviétique en Transition vers l’Économie de Marché.

Tableau 2. Emissions de gaz à effet de serre en 1990 (adapté de Kandel, 2002)

\begin{tabular}{lllll}
\hline & $\begin{array}{l}\text { MtC Eq. } \\
2000\end{array}$ & $\begin{array}{l}\text { \% du total } \\
\text { mondial } \\
2000\end{array}$ & $\begin{array}{l}\text { tC/hab } \\
2000\end{array}$ & $\begin{array}{l}\text { \% des } \\
\text { émissions } \\
1850-2000^{*}\end{array}$ \\
\hline Etats-Unis & 1892 & 20,6 & 6,6 & 29,8 \\
\hline Chine & 1356 & 14,8 & 1,1 & 7,3 \\
\hline Union Europ. (25) & 1283 & 14 & 2,8 & 27,2 \\
\hline Russie & 520 & 5,7 & 3,6 & 8,3 \\
\hline Inde & 506 & 5,5 & 0,5 & 2 \\
\hline Japon & 364 & 4 & 2,9 & 4,1 \\
\hline Allemagne & 265 & 2,9 & 3,2 & 7,5 \\
\hline Brésil & 230 & 2,5 & 1,3 & 1 \\
\hline Canada & 195 & 2,1 & 6,3 & 2,1 \\
\hline Royaume-Uni & 181 & 2 & 3,1 & 6,5 \\
\hline France & 137 & 1,5 & 2,3 & 3 \\
\hline Pays développés & 4776 & 52 & 3,9 & 77 \\
\hline Pays en développement & 4408 & 48 & 0,9 & 23 \\
\hline
\end{tabular}

Tableau 3. Emissions de gaz à effet de serre en 2000 et pourcentage des émissions de CO2 entre 1850 et 2000

$\mathrm{MtC}$ Eq : millions de tonnes d'équivalent Carbone ; $\mathrm{tC} / \mathrm{hab}$ : tonne de carbone par habitant

* pourcentage des émissions totales de $\mathrm{CO}_{2}$ d'origine fossile sur la période 1850-2000

Source : Barral, 2006 
L'analyse de l'évolution des émissions des pays de l'Annexe I fournit des renseignements intéressants sur les efforts en cours en vue du respect des engagements de ces pays. Entre 1990 et 2005, ces émissions ont baissé de 2,8\% en moyenne globale, passant de 18709 à $18181 \mathrm{Tg}$ d'équivalent $\mathrm{CO}_{2}$ (UNFCCC, 2007) ${ }^{7}$. Si la réduction est importante pour des pays d'Europe de l'Est, elle l'est beaucoup moins pour d'autres pays comme la Turquie, l'Espagne, le Portugal (fig. 2).

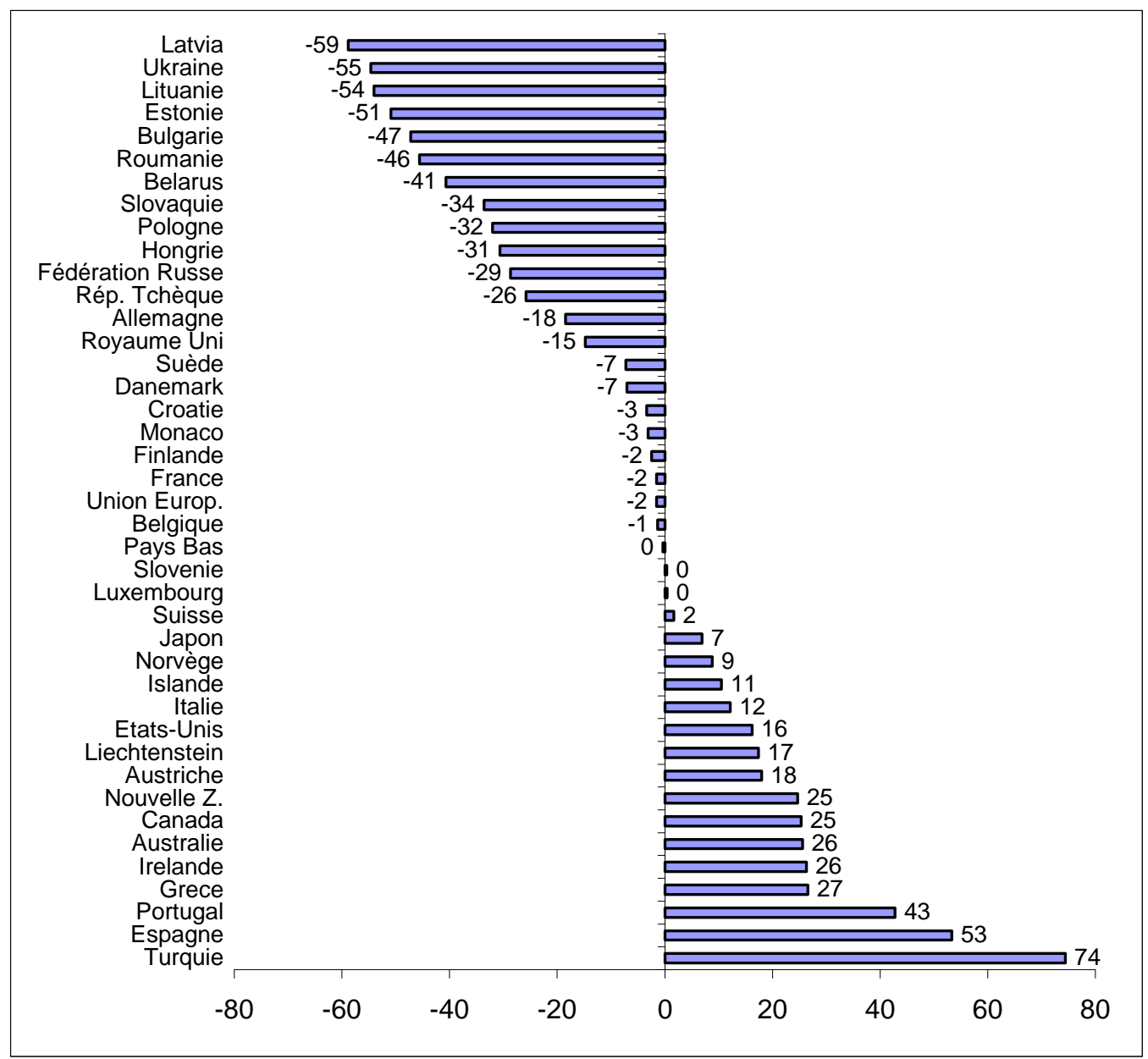

Figure 2. Evolution des émissions de gaz à effet de serre des pays de l'annexe I entre 1990 et 2005 (en \%, d'après les données UNFCCC, 2007)

La tendance moyenne globale vers la baisse entre 1990 et 2005 semble ralentir, la comparaison des émissions entre 2000 et 2005 révélant une hausse de $2,6 \%$ (UNFCCC, 2007). Cette tendance récente à la hausse, si elle se confirme et se poursuit, amène à se demander si l'objectif de réduction de $5 \%$ entre 2008 et 2012 sera atteint. Il y a lieu de s'interroger sur la pertinence et l'efficacité de cet objectif de réduction. Il n'est exigé qu'aux pays de l'Annexe I et seuls les pays de cet Annexe I qui ont ratifié le protocole sont tenus de respecter leurs engagements de réduction des émissions. Les émissions des pays de l'Annexe I représentent globalement la moitié des émissions mondiales. Bien que faisant partie des pays de cet Annexe I, les Etats-Unis n'ont pas ratifié le protocole et ne sont donc tenus pour l'instant à aucun effort contraignant de réduction de leurs émissions.

Par ailleurs, on note depuis quelques années une hausse importante des émissions des pays en développement et surtout des pays émergents dont la croissance économique et l'industrialisation sont sources d'une rapide augmentation de leurs productions de gaz à effet de serre (Jancovici, 2007 ;

\footnotetext{
${ }^{7} 1 \mathrm{Tg}$ (terragramme) représente 1 million de tonnes. Les statistiques citées dans ce paragraphe et dans la figure 2 ne prennent pas en compte les émissions-absorptions dans le secteur de l'utilisation des terres, du changement d'affectation des terres et de la foresterie (UTCATF dans le jargon onusien). Les gaz à effet de serre pris en compte sont le $\mathrm{CO}_{2}$, le $\mathrm{CH}_{4}$, le $\mathrm{N}_{2} \mathrm{O}$, les $\mathrm{HFC}$, les PFC et le $\mathrm{SF}_{6}$
} 
Tabeaud, 2008) ${ }^{8}$. Les données relatives au seul $\mathrm{CO}_{2}$ confirment cette augmentation des émissions totales des pays en développement et des pays émergents. En valeur absolue en 2005, les Etats-Unis sont le premier producteur mondial de $\mathrm{CO}_{2}$ (fig. 3). La production états-unienne constitue $67 \%$ de la production mondiale, devant celles de la Chine (5\%), de l'Italie (5\%) et de la France (4\%). D'après les prévisions, les émissions de $\mathrm{CO}_{2}$ des pays de l'Annexe I seront inférieures à celles des autres pays dans moins de 10 ans (Jancovici, 2007). La répartition par pays et par habitant parait corroborer le clivage Nord-Sud mais souligne surtout l'importance des pays du golfe, peu peuplés et producteurs de pétrole (fig. 4). Le Qatar produit en moyenne 69 tonnes de $\mathrm{CO}_{2}$ par habitant en 2005, le Koweït 38 et les Emirats Arabes Unis 37 (CDIAC, 2007). Cette moyenne est de 20 pour les Etats-Unis.

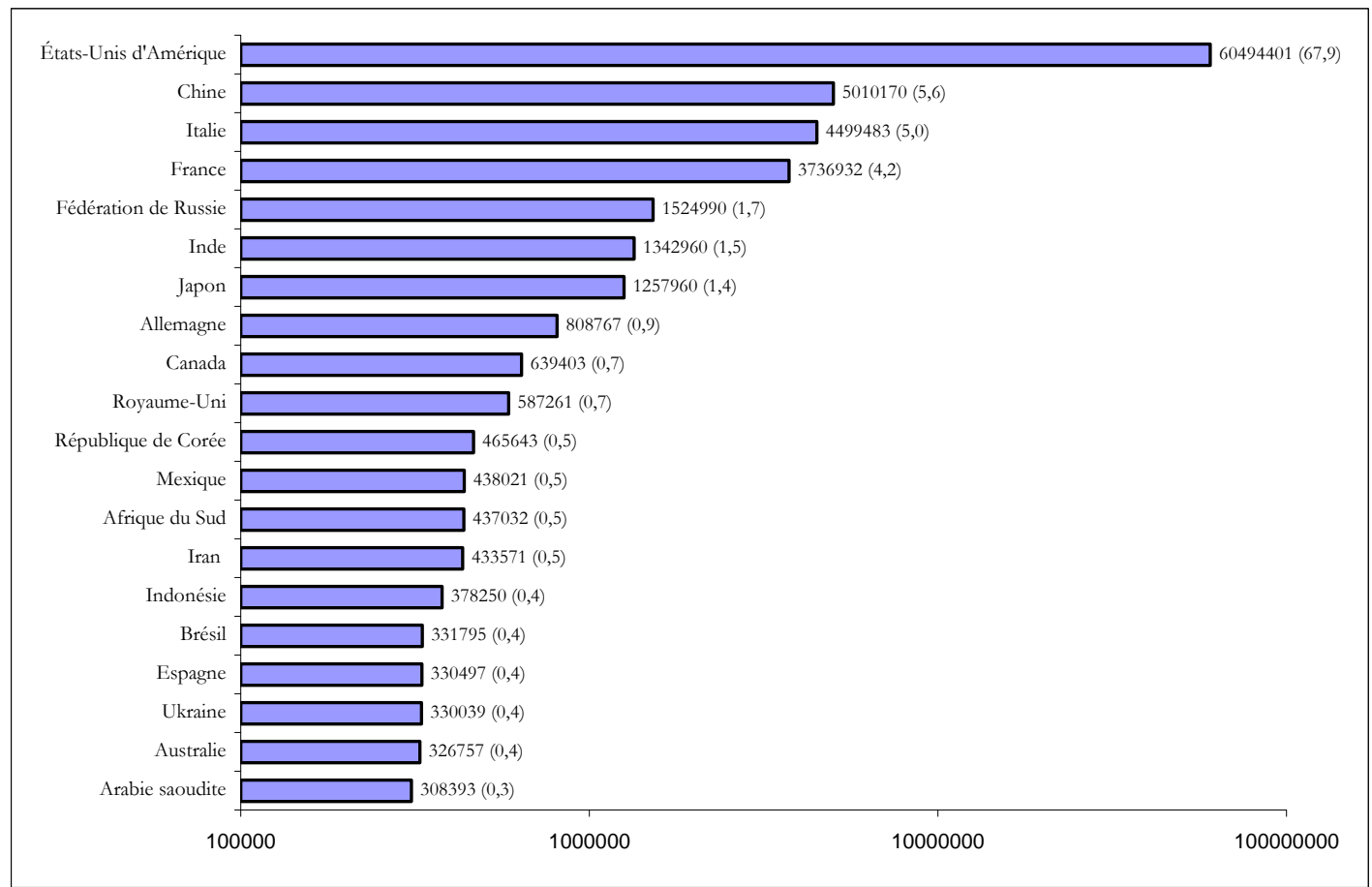

Figure 3. Les 20 plus grands émetteurs de $\mathrm{CO}_{2}$ en 2004 (milliers de tonnes et pourcentage, d'après les données UNFCCC, 2007)

La 《responsabilité historique » des pays développés pourra devenir caduque dans les années qui viennent si les émissions des pays en développement et des pays émergents continuent d'augmenter. Rien ne laisse envisager une réduction de ces émissions. Il apparaît vraisemblable que le respect strict du protocole de Kyoto, tout au moins pour la première phase (2008-2012), n'engendrera pas les effets escomptés en terme de baisse de la production mondiale des gaz à effet de serre. L'effet attendu sur la mitigation ou l'atténuation du réchauffement de la Terre n'est donc pas évident. De nombreuses études concluent à l'insuffisance et au caractère peu ambitieux du protocole de Kyoto (Durand, 2007).

Afin de revigorer la lutte contre le changement climatique en adoptant des politiques nationales et internationales beaucoup plus ambitieuses, des discussions et des négociations ont été engagées depuis $2005^{9}$ pour élaborer et adopter un traité qui remplacera le protocole de Kyoto après 2012. Les négociations visent d'une part à obtenir des pays développés l'engagement de réduire davantage leurs émissions de gaz à effet. D'autre part, ces négociations visent à impliquer davantage les pays en développement, et surtout les pays émergents, dans les efforts à fournir pour réduire le réchauffement de la Terre.

\footnotetext{
${ }^{8}$ Les émissions totales de la Chine ont augmenté de $152 \%$ entre 1990 et 2007 (Alternatives Internationales, 2008); par habitant, cette augmentation est de $98 \%$ durant cette même période (Tabeaud, 2008).

${ }^{9}$ A la suite de l'entrée en vigueur du protocole de Kyoto et surtout au cours de la conférence de Montréal (COP 11, c'est-àdire $11^{\text {ème }}$ conférence des parties ; ce type de conférence rassemble annuellement les Etats qui ont ratifié la convention climat afin de faire le point sur la mise en œuvre de la convention associée au protocole de Kyoto)
} 


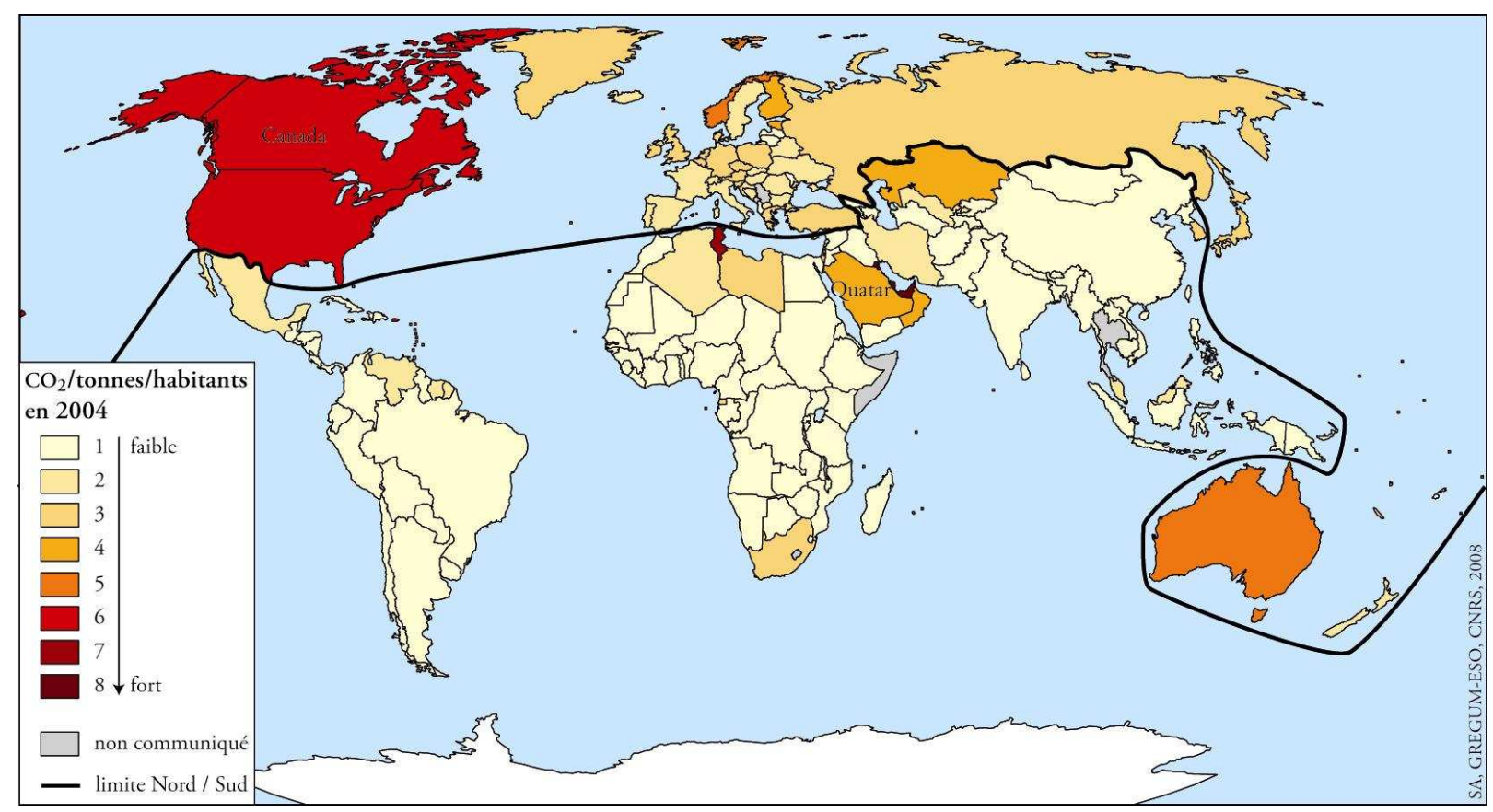

Figure 4. Emissions de $\mathrm{CO}_{2}$ par pays et par habitant en 2004 (d'après les données UNFCCC, 2007)

Les pays de l'Union européenne ont adopté le 12 décembre 2008 à Bruxelles, lors du sommet européen clôturant la présidence française du Conseil de l'Union, un accord pour la réduction de $20 \%$ des émissions de gaz à effet de serre des pays membres de l'Union à l'horizon $2020^{10}$. Un jour après, les pays qui ont ratifié la convention climat et le protocole de Kyoto, réunis à Poznan en Pologne, ont adopté une "feuille de route " pour la suite des négociations qui doivent déboucher sur un nouveau traité dont l'adoption est prévue pour décembre 2009 à Copenhague ${ }^{11}$. Cette feuille de route a été adoptée faute d'un compromis pour un accord entre les pays développés, les pays émergents et les pays en développement. Les pays en développement demandent aux pays développés d'alimenter un fonds international qui aidera les pays en développement à s'adapter et à faire face aux effets du changement climatique. Les pays émergents refusent de prendre des engagements de réduction de leurs émissions de gaz à effet de serre avant 2020 et demandent que les pays développés réduisent davantage leurs émissions. Certains pays émergents, comme la Chine, l'Inde ou le Brésil, indiquent qu'ils pourraient réduire leurs émissions seulement à partir de la période 2020-2030, voire 2050, si les pays développés réduisent leurs émissions d'au moins $50 \%$ d'ici $2020^{12}$. La pression s'exerce sur les pays émergents pour qu'ils s'engagent à réduire leurs émissions durant la période post-kyoto ou Kyoto II, à partir de 2013. Les pays émergents les plus ciblés par cette pression sont la Chine (en raison de l'importante hausse de ses émissions) et le Brésil (en raison de l'ampleur de la déforestation qui représente $75 \%$ de ses émissions).

\section{Développement et forte pression environnementale au Nord, sous-développement et faible pression environnementale au Sud}

L'utilisation des indicateurs, même s'ils ne permettent pas de saisir toute la réalité et la complexité d'un phénomène, permet d'avoir un aperçu ou une représentation de cette réalité. Les indicateurs servent par ailleurs à classer les pays et à les comparer entre eux, ce qui présente un intérêt pédagogique pour la compréhension des rapports entre pays ou entre groupes de pays, au-delà des critiques dont ces indicateurs peuvent être l'objet (Theys, 2001 ; Gadrey et al., 2003 ; Arnould et Vincent, 2004 ; Boisvert, 2005). Pour comparer les liens entre niveaux de développement et pressions

\footnotetext{
${ }^{10}$ Voir Le Monde, éditions du 13 et du 14 décembre 2008

${ }^{11}$ Voir Le Monde, éditions du 13 et du 14 décembre 2008

${ }^{12}$ Voir le dossier « réchauffement climatique : que vont faire les pays émergents ? », Alternatives Internationales, décembre 2008 .
} 
sur l'environnement dans une perspective Nord-Sud, nous avons choisi de faire appel à l'Indice de Développement Humain (IDH) et à l'Empreinte Ecologique (EE).

L'IDH a été élaboré en 1990 sous les auspices du Programme des Nations Unies pour le Développement (PNUD). Il fournit un aperçu du niveau de développement en prenant en compte non seulement la richesse économique des pays, mais aussi les paramètres " humains » ou "sociaux ». C'est pour cela que nous le préférons aux autres indicateurs de développement comme le PIB (Produit Intérieur Brut) ou le PNB (Produit National Brut) qui ont été conçus par les institutions financières internationales (Banque Mondiale et Fonds Monétaire International) et qui considèrent le développement essentiellement sous l'angle de la croissance économique et des revenus (Gadrey et al., 2003). L'IDH est construit à partir de 3 variables de base qui sont ensuite synthétisés : l'espérance de vie à la naissance associée à l'accès de la population aux soins, l'alphabétisation des adultes associée au taux de scolarisation combiné pour l'enseignement primaire, secondaire et supérieur, et le PIB corrigé par la parité des pouvoirs d'achat en dollars US. L'IDH est un indicateur composite exprimé sur une échelle allant de 0 (pays à développement humain faible) à 1 (pays à développement humain élevé). Il caractérise le développement vu comme la capacité de mener une vie longue et saine avec accès à la connaissance et à l'information en plus de disposer de revenus ou de richesse permettant un niveau décent de vie.

Les données les plus récentes de l'IDH datent de 2005 et distinguent 3 catégories de pays (PNUD, 2007): les pays à développement humain élevé (IDH égal ou supérieur à 0,800 ), les pays à développement humain médian (IDH compris entre 0,500 et 0,799 ) et les pays à faible développement humain (IDH inférieur à 0,500 ). La moyenne mondiale de l'IDH est de 0,743 en 2005 pour un total de 177 pays. Pour les pays à développement humain élevé, la moyenne est de 0,897 , alors qu'elle est de 0,698 pour les pays à développement humain moyen et 0,436 pour les pays à développement humain faible (fig. 5). La catégorie des pays à faible développement humain (22 pays) est constituée uniquement de pays d'Afrique. Beaucoup de pays du Sud (Brésil, Argentine, Chili, Mexique, Libye) appartiennent à la catégorie des pays à développement humain élevé, ce qui rend moins pertinent le découpage Nord-Sud issu de la convention climat et repris par le protocole de Kyoto. Puisque les efforts à fournir par les pays en matière de lutte contre le changement climatique ont été définis sur la base du niveau de développement, il ne parait pas aberrant de solliciter les pays du Sud qui présentent des niveaux de développement comparables à ceux des pays du Nord. La remise en cause du découpage Nord-Sud au regard de l'IDH vaut aussi au regard de l'empreinte écologique.

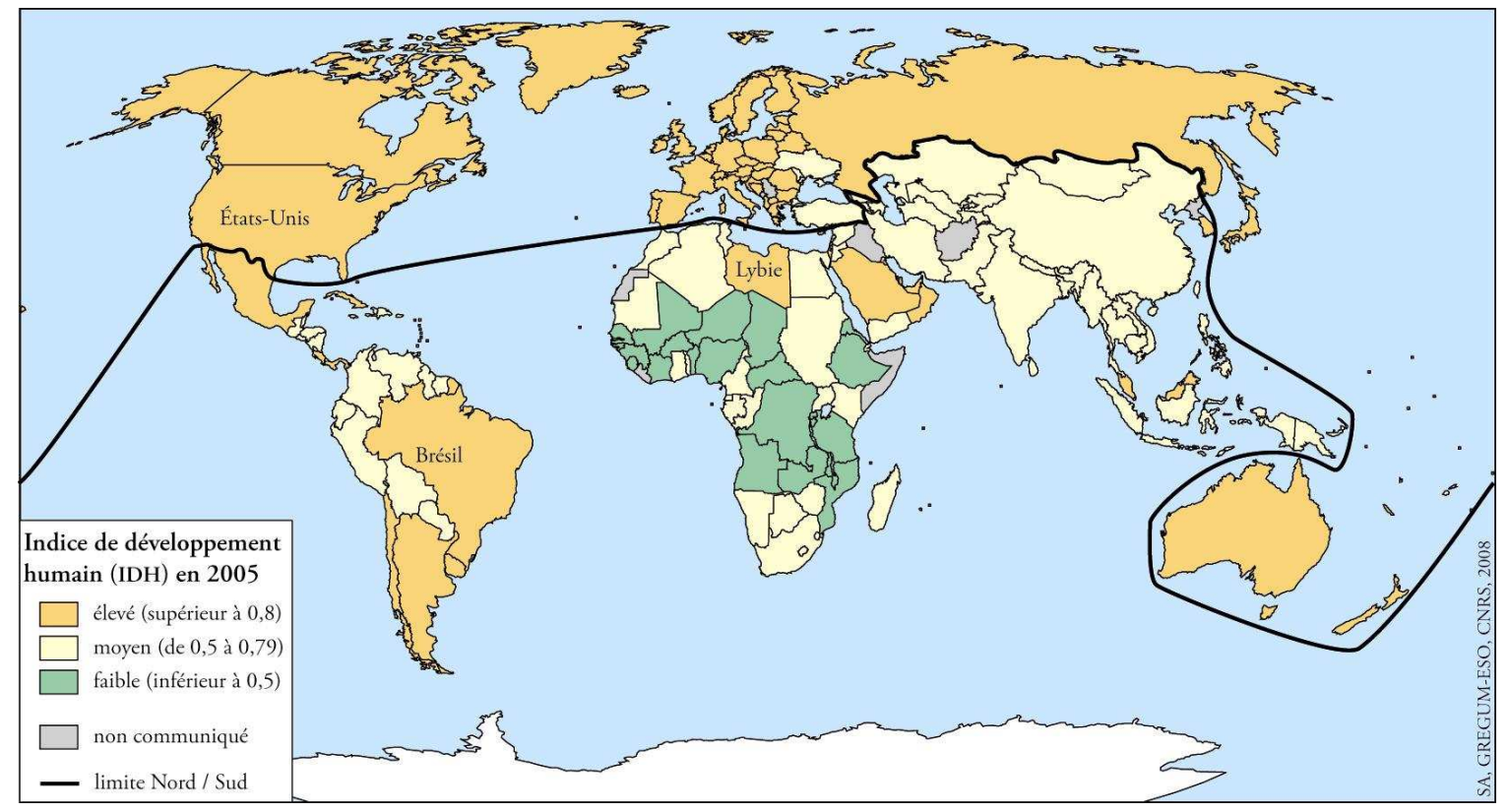

Figure 5. Répartition des pays par niveaux de développement humain en 2005 (d'après les données du PNUD, 2007) 
L'empreinte écologique (EE) est un indicateur d'environnement issu des travaux de Wackernagel et Rees (1999). Ce n'est pas un indicateur de développement durable puisqu'il permet d'évaluer seulement la composante écologique ou environnementale du développement durable. Pour une évaluation non partielle du développement durable, il est indispensable de compléter ou de coupler l'EE avec les indicateurs économiques et sociaux comme l'IDH. L'EE est défini comme étant la surface totale nécessaire d'une part à la production de la nourriture et des fibres, et d'autre part à l'absorption des déchets provenant de la consommation d'énergie. Il mesure les besoins d'utilisation de l'espace et des ressources naturelles par les sociétés humaines et confronte ces besoins avec la capacité de ces espaces à supporter l'utilisation anthropique et à se renouveler. Ainsi, l'EE quantifie la pression que l'Homme exerce sur la Terre en évaluant la surface productive nécessaire à l'humanité pour satisfaire sa demande de consommation en ressources et pour absorber les déchets issus de cette consommation de ressources.

En évaluant ce qui est demandé à l'environnement considéré globalement par rapport à ce que cet environnement global peut offrir sans perdre totalement son potentiel, l'EE se démarque des indicateurs qui n'évaluent que les prélèvements environnementaux effectués par les sociétés : nombre d'espèces disparues, listes rouges de l'UICN, taux d'épuisement des ressources en eau, degré d'artificialisation des milieux, ...(IFEN, 2001 et 2003; Ayong Le Kama et al., 2004). L'environnement global dont il est question dans l'EE est une agrégation de 6 domaines principaux : agriculture, élevage, forêt, pêche, infrastructure et combustibles fossiles. Les besoins sont pris en compte et mesurés dans ces principaux domaines et les résultats, par pays et par habitant, sont exprimés en hectares globaux (hg). Ainsi conçu, cet indicateur nous parait pertinent pour renseigner sur la pression exercée sur les ressources environnementales dans le monde. L'EE a été vulgarisé par le WWF qui l'utilise depuis 1998 comme matière première pour le rapport " planète vivante ». Ce rapport établit un classement des pays et offre un aperçu de l'environnement tel qu'il est sollicité pour la satisfaction des besoins de la population (EE) en comparaison avec les disponibilités environnementales «naturelles » (biocapacité).

Les dernières statistiques sur l'EE des pays ont été publiées en 2008 et rendent compte des mesures relatives à la situation de l'année 2005 (WWF, 2008) ${ }^{13}$. D'après ces données, l'EE mondiale était de 17,5 milliards d'hectares globaux, soit en moyenne $2,3 \mathrm{hg} /$ personne, alors que la biocapacité mondiale était de 13,6 milliards d'hectares globaux, soit en moyenne 2,1 hg/personne. La demande mondiale en ressources vivantes procurée par la Terre est donc supérieure de près de $30 \%$ à la capacité d'offre et de régénération de la Terre. L'EE d'un habitant des pays développés est plus de 6 fois supérieure à celle d'un habitant des pays en développement. L'empreinte moyenne d'un habitant des Etats-Unis est de 9,4 hg, alors que celle d'un habitant de la France est de 4,9 hg (fig. 6).

Comme pour l'IDH ou l'émission du $\mathrm{CO}_{2}$, des pays du Sud ont une empreinte écologique par habitant qui approche celle de certains pays du Nord. C'est le cas des Emirats Arabes Unis (avec une EE de 9,5 $\mathrm{hg} /$ personne, la valeur la plus élevée) ou du Koweït (avec EE de 8,9 hg/personne). L'empreinte dans plusieurs pays d'Amérique latine (Uruguay, Mexique, Chili), d'Asie Pacifique (Singapour, République de Corée, Mongolie), d'Afrique (Libye, Namibie, Botswana), du Moyen Orient et d'Asie Centrale (Israël, Oman, Turkménistan) est du même niveau que celle de la plupart des pays d'Europe du Nord et de l'Est (fig. 6).

\footnotetext{
${ }^{13}$ Outre l'EE, le WWF propose dans ce rapport un calcul de l'Empreinte Eau de Production 1997-2001.
} 


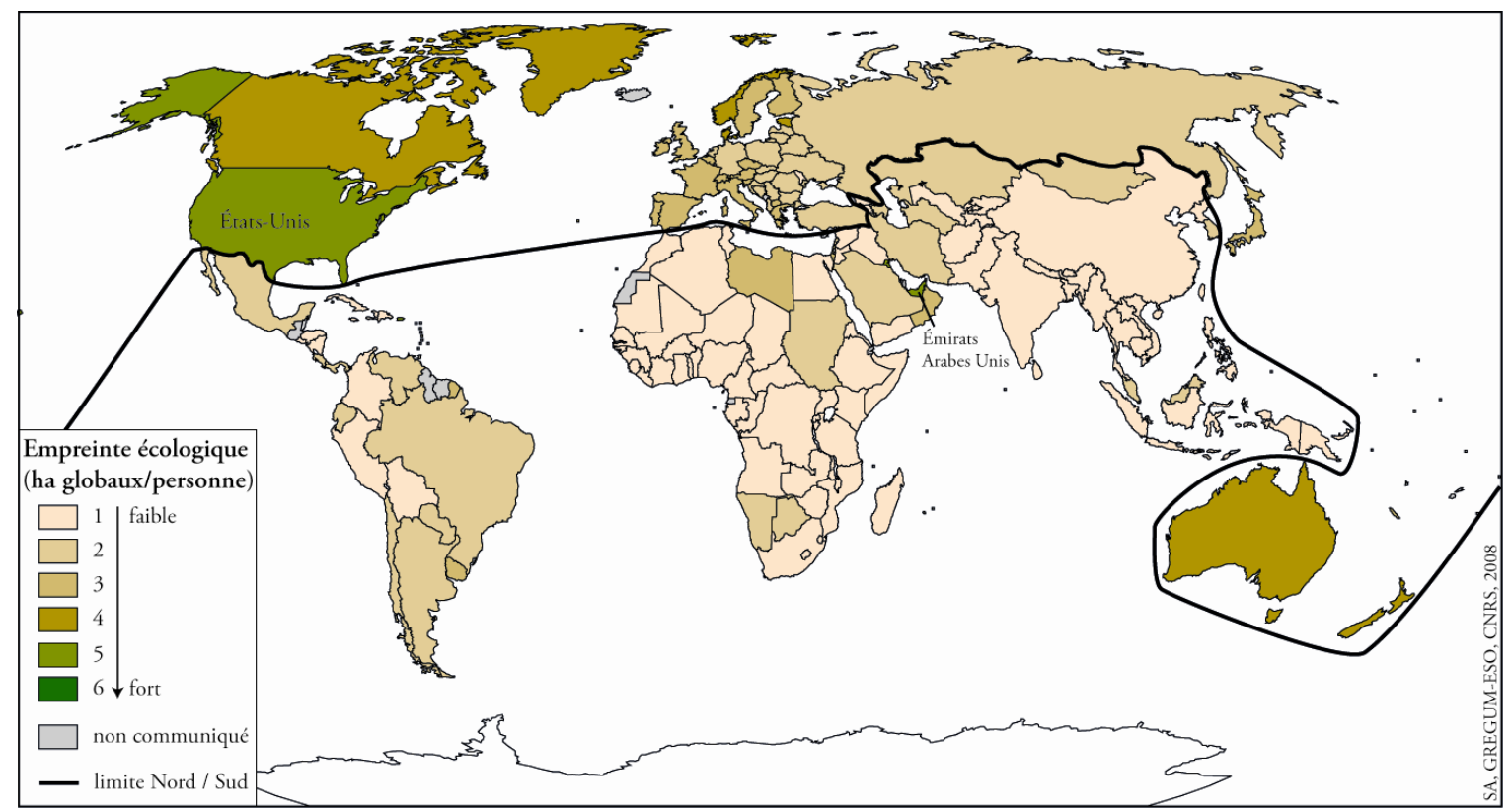

Figure 6. Répartition des pays suivant l'empreinte écologique en 2005 (d'après les données du WWF, 2008)

La comparaison des données de l'IDH et de l'EE (fig. 5 et 6) conforte globalement le clivage NordSud et l'idée que le développement génère une plus grande susceptibilité à la dégradation de l'environnement. Il ne parait donc pas exagéré d'affirmer que le développement est synonyme d'amenuisement des ressources ou de pressions environnementales. Riches ou développés, les pays du Nord sont en situation de déficit écologique (empreinte écologique supérieure à la biocapacité). Pauvres avec un faible développement économique et social, les pays du Sud sont de manière générale en situation d'excédent écologique (empreinte écologique inférieure à la biocapacité) ${ }^{14}$. Ces observations vont aussi bien dans le sens des pays du Nord soucieux des problèmes d'environnement que dans celui des pays du Sud davantage préoccupés par la lutte contre la pauvreté. Or en se préoccupant de l'environnement, les pays du Nord donnent l'impression de négliger la lutte contre la pauvreté. Parallèlement, les pays du Sud donnent l'impression de négliger l'environnement lorsqu'ils se préoccupent de la pauvreté. Dans ce contexte, comment dépasser le clivage Nord-Sud et la dichotomie entre développement et environnement, afin de pouvoir impliquer les pays du Nord et ceux du Sud, en conciliant la lutte contre la dégradation de l'environnement et la lutte contre la pauvreté et le sous-développement?

\section{Sortir du clivage Nord-Sud et de l'opposition entre développement et environnement : le challenge du développement durable}

Le développement durable a émergé dès 1987 suite à la publication du rapport de la Commission Mondiale sur l'Environnement et le Développement (Commission Brundtland créée en 1983 par l'ONU). La Conférence des Nations Unies sur l'Environnement et le Développement, tenue en 1992 à Rio de Janeiro au Brésil, a ensuite consacré le développement durable comme leitmotiv planétaire en amenant la communauté internationale à prendre conscience de l'ampleur et de l'enjeu des problèmes d'environnement et de développement dans le monde. Le développement durable a depuis lors pris une place considérable dans les discours et les politiques nationales et internationales (Jollivet, 2001 ; Aubertin et Vivien, 2006 ; Smouts, 2005 ; Froger, 2006 ; Mancebo, 2006 ; Veyret, 2007). Il est devenu un aspect important de la géopolitique mondiale car il s'agit d'obtenir de l'ensemble des États du

\footnotetext{
${ }^{14} \mathrm{Il}$ convient néanmoins de nuancer ces observations dans la mesure où la situation de certains pays du Sud, en particulier les pays émergents d'Amérique du sud ou d'Asie Pacifique, du Moyen Orient et d'Asie Centrale, tant en ce qui concerne l'IDH que l'EE, évolue et devient de plus en plus proche de celle des pays développés. Entre 2003 et 2005, l'EE par habitant a augmenté essentiellement dans les pays du Sud, l'Afrique ayant connu la plus grande hausse (21\%), alors que l'EE a très sensiblement baissé dans les pays du Nord durant le même laps de temps (WWF, 2006 et 2008).
} 
monde l'engagement de mettre en œuvre des politiques pour « un développement qui soit efficace économiquement tout en étant socialement équitable et écologiquement supportable ». Le développement durable insiste sur la conciliation entre environnement et économie, étant entendu que la croissance économique devrait améliorer le niveau de vie et ne pas se faire au détriment de l'environnement. Ainsi conçu, le développement durable peut être appréhendé ou évalué de manière pédagogique en utilisant les indicateurs environnementaux comme l'EE, et les indicateurs économiques et sociaux comme l’IDH.

Le croisement de l'IDH et de l'EE dans une représentation graphique permet d'illustrer la situation des pays au regard des exigences du développement durable en partant de l'hypothèse que le développement durable est synonyme d'un IDH élevé et d'une EE faible. Pour cet exercice, nous considérons qu'un pays est réputé avoir un développement économique et social satisfaisant si son IDH en 2005 est supérieur ou égal à 0,8 (seuil de développement économique et social correspondant au seuil de développement élevé d'après le rapport 2007 du PNUD). Nous considérons en outre que l'environnement d'un pays est réputé non dégradé irréversiblement si l'EE de ce pays en 2005 est inférieure ou égale à $2,1 \mathrm{hg} / \mathrm{hab}$ (seuil de durabilité écologique équivalente à la biocapacité moyenne mondiale d'après le rapport $2008 \mathrm{du}$ WWF). L'utilisation de ces deux indicateurs suppose d'admettre que le développement durable signifie pour un pays que son IDH soit supérieur ou égal à 0,8 et que son EE soit inférieure ou égale à 2,1 hg/hab. Seuls Cuba (avec une EE de 1,8 hg/hab et un IDH de 0,838 ) et Trinidad et Tobago (avec une EE de 2,1hg/hab et un IDH de 0,814) remplissent cette double exigence (fig. 7). Des études précédentes basées sur les données de 2000 ont montré qu'aucun pays ne remplissait ces critères (Boutaud et al., 2002 ; Boutaud, 2004 et 2008). Les données de 2003 indiquent que Seul Cuba remplissait ces critères (WWF, 2006).

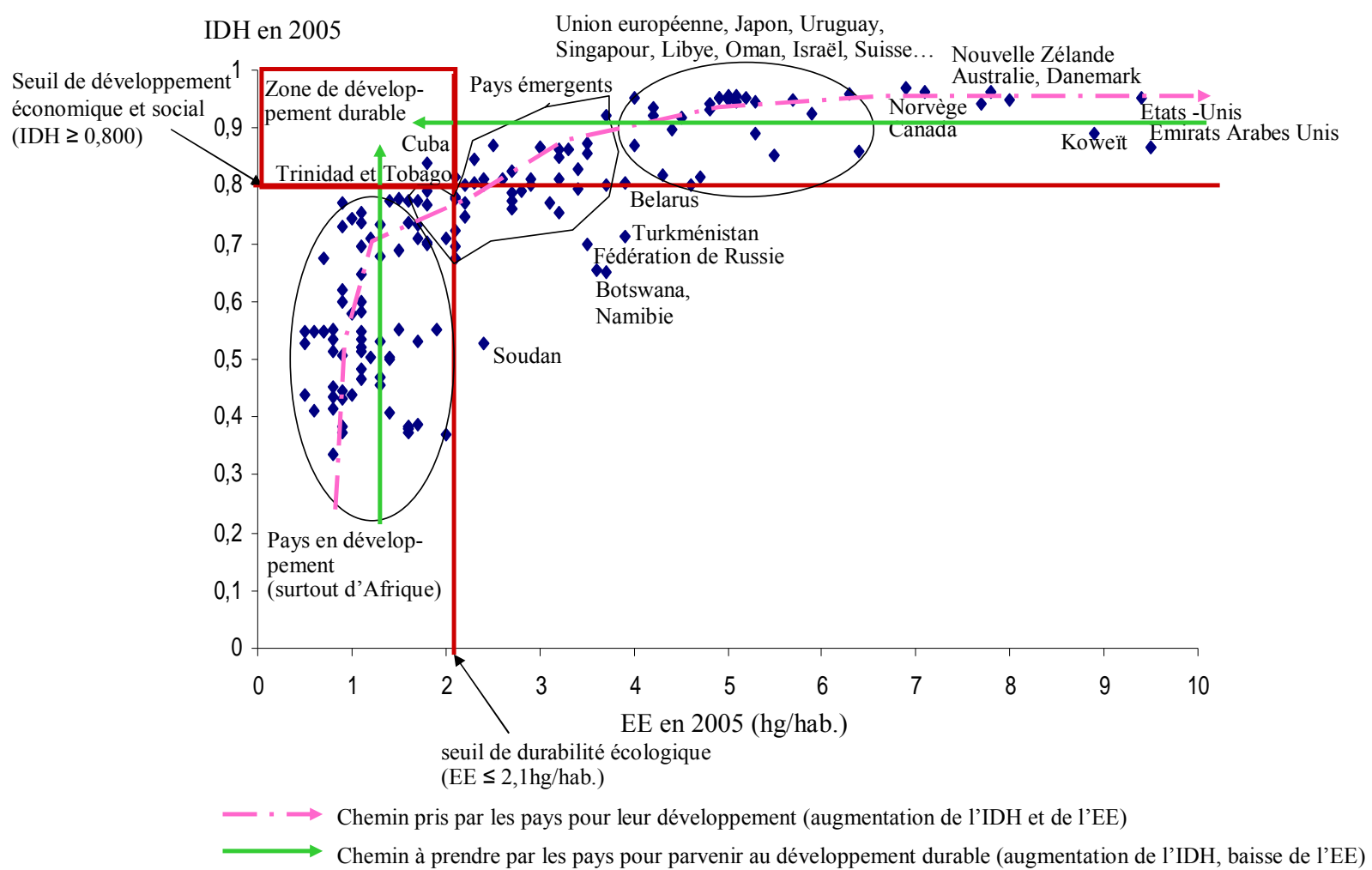

Figure 7. Représentation croisée de l'IDH en 2005 et de l'EE en 2005 pour situer les pays par rapport aux exigences du développement durable (d'après les données du PNUD, 2007 pour l'IDH et du WWF, 2008 pour l'EE)

Lorsque l'IDH d'un pays est supérieur à 0,8 , son EE est généralement très élevée. Cette situation caractérise la quasi-totalité des pays développés et indique que le développement est globalement synonyme de dégradation de l'environnement par le dépassement de la biocapacité (déficit 
écologique). Lorsque l'EE d'un pays est inférieure ou égale à $2,1 \mathrm{hg} / \mathrm{hab}$, son IDH est généralement inférieur au seuil de 0,8 . Ce constat concerne surtout les pays d'Afrique subsaharienne et suggère que l'absence de développement économique et social est globalement synonyme de conservation de l'environnement (excédent écologique). L'augmentation de l'IDH d'un pays, lors de son développement, entraîne l'augmentation de son EE. L'EE de certains pays en développement ou en émergence semble très disproportionné par rapport à leur IDH. C'est le cas par exemple des Emirats Arabes Unis, du Koweït et de l'Estonie qui ont une EE de type pays développés alors que leur IDH est tout juste de l'ordre de $0,8^{15}$. Ces données invitent une fois de plus à atténuer le clivage Nord-Sud qui situe les Emirats Arabes Unis et le Koweït parmi les pays du Sud auxquels la convention climat et le protocole de Kyoto n'exigent pas la réduction des émissions de gaz à effet de serre.

Pour parvenir au développement durable, les pays développés devraient réduire leur EE tout en maintenant leur IDH élevé alors que les pays en développement devraient maintenir leur faible EE et augmenter leur IDH (fig. 7). Il s'agit donc, dans un cas comme dans l'autre, d'une double exigence. Les pays qui ne sont pas loin de satisfaire à cette double exigence sont des pays qui ont un IDH proche de 0,8 et une empreinte écologique proche de $2,1 \mathrm{hg} / \mathrm{hab}$. Nous avons retenu les seuils de 0,743 pour l'IDH (moyenne mondiale en 2005 d'après le PNUD) et de 2,7 hg/hab pour l'EE (moyenne mondiale en 2005 d'après le WWF) pour établir cette liste de pays (classés par ordre alphabétique) que nous appelons pays émergents dans la perspective du développement durable (tabl. 4).

\begin{tabular}{lrr}
\hline Pays & EE 2005 & IDH 2005 \\
\hline Albanie & 2,2 & 0,801 \\
\hline Arabie Saoudite & 2,6 & 0,812 \\
\hline Argentine & 2,5 & 0,869 \\
\hline Arménie & 1,4 & 0,775 \\
\hline Azerbaïdjan & 2,2 & 0,746 \\
\hline Brésil & 2,4 & 0,8 \\
\hline Bulgarie & 2,7 & 0,824 \\
\hline Chine & 2,1 & 0,777 \\
\hline Colombie & 1,8 & 0,791 \\
\hline Costa Rica & 2,3 & 0,846 \\
\hline Equateur & 2,2 & 0,772 \\
\hline Géorgie & 1,1 & 0,754 \\
\hline Iran & 2,7 & 0,759 \\
\hline Jordanie & 1,7 & 0,773 \\
\hline Malaisie & 2,4 & 0,811 \\
\hline Maurice & 2,3 & 0,804 \\
\hline Pérou & 1,6 & 0,773 \\
\hline Philippines & 0,9 & 0,771 \\
\hline Rép. Dominicaine & 1,5 & 0,779 \\
\hline Sri Lanka & 1 & 0,743 \\
\hline Thaillande & 2,1 & 0,781 \\
\hline Tunisie & 1,8 & 0,766 \\
\hline Turquie & 2,7 & 0,775 \\
\hline Ukraine & 2,7 & 0,788 \\
\hline Venezuela & 2,8 & 0,792 \\
\hline & &
\end{tabular}

Tableau 4. Pays émergents dans la perspective du développement durable (d'après les données du PNUD et du WWF)

Les données représentées dans la figure 7 indiquent que les chemins à emprunter pour satisfaire aux exigences du développement durable sont différents selon qu'il s'agit des pays développés ou des pays

\footnotetext{
${ }^{15}$ Les Emirats Arabes Unis ont une EE de 9,5 (la plus élevée de tous les pays) et un IDH de 0,868; le Koweït a une EE de 8,9 ( $3^{\text {ème }}$ mondiale après les Emirats et le Canada) et un IDH de 0,891 ; l'Estonie a une EE de 6,4 (supérieure à la moyenne de l'Union européenne) et un IDH de 0,860.
} 
en développement. Cette observation a surtout un intérêt pédagogique en lien avec la conception du développement durable fondée sur les seuils de l'IDH et de l'EE. Elle montre d'une part la différence entre développement et développement durable et d'autre part l'ampleur des efforts à fournir par les pays en vue de parvenir à ce développement durable. S. Latouche (2001) résume cette différence et ces efforts en faisant remarquer que «quand on est à Rome et que l'on doit se rendre par le train à Turin, si on s'est embarqué [...] dans la direction de Naples, il ne suffit pas de ralentir la locomotive, de freiner ou même de stopper, il faut descendre et prendre un autre train dans la direction opposée ; pour sauver la planète et assurer un futur acceptable à nos enfants, il ne faut pas seulement modérer les tendances actuelles, il faut carrément sortir du développement [...]» tel qu'il s'est réalisé jusqu'à présent. La figure 7 montre en effet que les pays développés et les pays en développement devraient changer les chemins ou les itinéraires pris par leur développement si ces pays veulent parvenir au développement durable, c'est-à-dire un développement économique et social qui ne se fasse pas au détriment de l'environnement.

\section{Conclusion}

Les données traitées et présentées dans cet article montrent que le développement des pays s'est réalisé et se réalise encore en générant la dégradation de l'environnement, comme si le développement et la protection de l'environnement sont antinomiques on contradictoires. Les pays développés figurent globalement aux premiers rangs des classements des pays suivant les quantités de gaz à effet de serre émises ou suivant l'empreinte écologique, même si quelques pays en développement, comme la Chine ou les Emirats Arabes Unis, apparaissent souvent et de plus en plus en tête de ces classements. En incorporant le clivage entre pays développés (pays de l'Annexe I) et pays en développement (reste du monde), la convention des Nations unies sur le changement climatique et le protocole de Kyoto ont admis l'opposition ou la contradiction entre développement et environnement. Ce clivage, sous-tendu par le principe de la «responsabilité historique» des pays développés dans le réchauffement de la Terre, est devenu un fondement primordial de la géopolitique internationale dans le cadre de la lutte contre le changement climatique. Il est utilisé par les pays en développement et les pays émergents comme principal argument pour rejeter les demandes de réduction de leurs émissions de gaz à effet de serre. De la sorte, ces pays en développement, associés aux pays émergents, continuent de demander aux pays développés d'être à l'avant-garde et de fournir l'essentiel de l'effort nécessaire pour lutter contre le changement climatique. Pour surpasser ce clivage, il est indispensable de chercher à concilier le développement et la protection de l'environnement. C'est l'ambition et l'objectif majeur du développement durable tel qu'il a été mis à l'ordre du jour de la communauté internationale par les Nations unies. L'analyse croisée des indicateurs économique (IDH) et écologique (EE) montre que cet objectif n'est pas facile à atteindre et qu'il suppose une modification profonde de la conception du développement pour parvenir au développement durable.

Pour que la lutte contre le changement climatique soit efficace au-delà de la première phase d'application du protocole de Kyoto, la communauté internationale devra être plus ambitieuse en s'engageant, pour l'après 2012, sur des objectifs chiffrés de réduction des gaz à effet de serre beaucoup plus élevés que l'objectif de 5\% adopté pour la période 2008-2012. Dans cette perspective, les négociations engagées depuis 2005 (conférence de Montréal) continuent d'exiger des efforts aux pays développés tout en cherchant à impliquer davantage les pays en développement et surtout les pays émergents. Des initiatives allant dans ce sens sont inscrites dans l'agenda des réunions internationales (Hall et al., 2008). Il s'agit notamment du Mécanisme pour un Développement Propre (MDP). Il permet aux pays développés de remplir une partie de leurs engagements de réduction de leurs émissions de gaz à effet de serre en finançant dans des pays en développement des projets à technologie peu productrice de gaz à effet de serre. Ainsi, les pays en développement se développent " proprement» (en émettant une quantité de gaz à effet de serre inférieure à la quantité qui aurait été émise en l'absence de la technologie "propre ») et les pays développés sont crédités de quotas d'émission de gaz à effet de serre correspondant au volume évité ou économisé grâce à leur technologie "propre». Les modalités de mise en œuvre de ce mécanisme, qui fait partie des mécanismes de flexibilité du protocole de Kyoto, ont été définies dans les accords de Marrakech en 2001. C'est depuis 2005, suite à la création des marchés internationaux de carbone (en particulier celui de la Banque Mondiale et celui de l'Union Européenne), que les projets MDP foisonnent dans les pays 
en développement et surtout dans les pays émergents (Barral, 2006 ; Denis, 2007 ; Höhlne et al., 2007 ; Tsayem, 2008b). D'autres initiatives de coopération Nord-Sud en matière de lutte contre le changement climatique et de promotion du développement durable sont en cours d'expérimentation suite aux décisions prises lors de la conférence de Bali en 2007. C'est le cas de la Déforestation et de la Dégradation Evitées qui est un mécanisme d'incitations financières internationales au profit des pays en développement qui acceptent d'appliquer des politiques conduisant à la baisse de la déforestation, celle-ci étant responsable de 20 à $25 \%$ des émissions mondiales de gaz à effet de serre (Karsenty et Pirard, 2007a et b). Le bilan de ces initiatives émergentes sera présenté et discuté lors de la conférence de Copenhague en décembre 2009, afin d'intégrer ou de renforcer éventuellement ces initiatives dans le prochain accord international pour la période post-Kyoto (à partir de 2013).

\section{Références bibliographiques}

Allègre C., 2007. Ma vérité sur la planète. Plon Fayard, 237 p.

Alternatives Internationales, $2008\left(n^{\circ} 41\right)$. Dossier Réchauffement climatique : que vont faire les pays émergents ? p.48-59.

Alternatives Sud, $2006\left(\mathrm{n}^{\circ} 2\right)$. Changements climatiques : impasses et perspectives. Points de vue du Sud. Centre Tricontinental, Syllepse, 209 p.

Arnould P., Clément V., 2004. Forêts et développement durable. De l'impératif de la durabilité à la question controversée des indicateurs. Historiens et Géographes n 387, p.163-174.

Arnould P., Simon L., 2007. Géographie de l'environnement. Belin, 304 p.

Aubertin C., Vivien F.D. (dir.), 2006. Le développement durable: enjeux politiques, économiques et sociaux. IRD Editions/La documentation Française, 143 p.

Ayong Le Kama A., Lagarenne C., Le Lourd P. (dir.), 2004. Indicateurs du développement durable : lesquels retenir ? Ministère de l'Ecologie et du Développement Durable, La documentation française, $236 \mathrm{p}$.

Barral J.P., 2006. Aider les pays du Sud à lutter contre leurs émissions de gaz à effet de serre : le mécanisme de développement propre prévu par le protocole de Kyoto est-il la solution? In A quoi sert d'aider le Sud? (directeur : Michaïlof S.), Economica, p. 271-297.

Boisvert V. 2005. L' « empreinte écologique » : un indicateur de développement durable ? In Le développement durable : une perspective pour le $21^{\text {ème }}$ siècle (directeurs : Maréchal J.-P. et Quenault B.), PUR, p.p. 165-183.

Boutaud A., 2004. Le développement durable : penser le changement ou changer le pansement ? Doctorat en Sciences de la Terre et de l'Environnement, Ecole des Mines de Saint Etienne \& Université Jean Monnet.

Boutaud A., 2008. Education au développement durable (http://www.alsace.iufm.fr/web/ressourc/pedago/discipli/svt/EDD trame formation 2008 1.pdf).

Boutaud A., Brodhac C., Gondran N., 2002. Quand le développement perd le Nord! Courbes de Kuznets environnementales : l'apport des indicateurs alternatifs de type empreinte écologique dans la réflexion sur le développement durable. Communication présentée au colloque Développement durable : leçons et perspectives. Ouagadougou 1-4 juin 2004. Agence universitaire de la Francophonie, Agence intergouvernementale de la Francophonie et Université de Ouagadougou (http://www.francophonie-durable.org).

Capedepuy V., 2007. La limite Nord/Sud. Mappemonde, $\mathrm{n}^{\circ} 88-4$.

Carroué L.(dir.), 2006. Globalisation, mondialisation. Historiens et Géographes, n³95, p.81-220.

CDIAC, 2007. On line trends. A compendium of data on global change (http://cdiac.ornl.gov/trends/trends.htm).

Da Lage A., Amat J.-P., Frérot A.-M., Guichard-Anguis S., Laferrière B.-J., Wicherek S.-P., 2008. L'après développement durable. Espaces, nature, culture et qualité. Ellipses, 351 p.

Denis B., 2007. Le mécanisme pour un développement propre. Un instrument politique au service du développement et de la préservation du climat? In Environnement et inégalités sociales (éds. Cornut P., Bauler T., Zaccaï E.), Editions de l'Université de Bruxelles, p. 157-170.

Durand F., 2007. Le réchauffement climatique en débats. Ellipses, 187 p.

Froger G. (dir.), 2006. La mondialisation contre le développement durable ? Ecopolis, PIE-Peter Lang, 315 p. Gadray J., Jany-Catrice F., Ribault T., Boidin B., 2003. Les indicateurs de richesse et de développement. Un bilan international en vue d'une initiative française. Rapport de recherche pour la DARES, $178 \mathrm{p}$.

GIEC, 2001. Changements climatiques 2001. Rapport de synthèse. Résumé à l'attention des décideurs, $37 \mathrm{p}$. (http://www.ipcc.ch/pdf/climate-changes-2001/synthesis-spm/synthesis-spm-fr.pdf).

GIEC, 2007. Changements climatiques 2007. Rapport de synthèse. Résumé à l'attention des décideurs, $114 \mathrm{p}$. (http://www.ipcc.ch/pdf/assessment-report/ar4/syr/ar4_syr_fr.pdf).

Hall D.S., Levi M., Pizer W.A., Ueno T., 2008. Policies for developing country engagement. Discussion paper 08-15, Harvard Kennedy School, The Harvard project on international climate agreements, 44 p. (www.belfercenter.org/climate). 
Höhne N., Wartmann S., Herold A., Freibauer A., 2007. The rules for land use, land use change and forestry under the Kyoto Protocol. Lessons learned for the future climate negotiations. Environmental Science and Policy, n¹0, p.353-369.

IFEN, 2001. Propositions d'indicateurs de développement durable pour la France. Etudes et Travaux, n³5, 106 p.

IFEN, 2003. 45 indicateurs de développement durable. Une contribution de l'IFEN. Etudes et Travaux, $\mathrm{n}^{\circ} 41$, $145 \mathrm{p}$.

Jancovici J.M., 2007. Comment évoluent actuellement les émissions de gaz à effet de serre ? (http://www.manicore.com/documentation/serre/GES.html).

Jollivet M. (éd.), 2001. Le développement durable, de l'utopie au concept. De nouveaux chantiers pour la recherche. Elsevier, 288 p.

Kandel R., 2002. Le réchauffement climatique : le grand risque. PUF, collection que sais-je ? 127 p.

Karsenty A., Pirard R., 2007a. Changement climatique : faut-il récompenser la « déforestation évitée »? Natures Sciences Sociétés, $\mathrm{n}^{\circ} 15$, p. 357-369.

Karsenty A., Pirard R., 2007b. Forêts tropicales : la question du bien public mondial et la quête d'instruments économiques multilatéraux pour un régime international. Revue Forestière Française, n 5, p. 535-547.

L’Information Géographique, 2007 (n³). Développement durable, 87 p.

Latouche S. 2001. Le développement peut-il être «durable»? L’Ecologiste, n 4, p.p. 38-39.

Le Monde, 2008. La Conférence de l'ONU avance, sans conclure, sur le financement. Les 27 trouvent un compromis sur le climat (éditions du 13 et du14 décembre).

Leroux M., 2002. «Global warming » : mythe ou réalité. L'évolution réelle de la dynamique du temps. Annales de Géographie, ${ }^{\circ} 624$, p.115-137.

Mancebo F., 2006. Le développement durable. A. Colin, 269 p.

Miossec A., Arnould P., Veyret Y., 2004. Vers une géographie du développement durable. Historiens et Géographes $\mathrm{n}^{\circ} 387, \mathrm{p} .81-260$.

PNUD, 2007. Rapport sur le développement humain dans le monde 2007/2008. La lutte contre le changement climatique : un impératif de solidarité humaine dans un monde divisé, $382 \mathrm{p}$.

Quenault B., 2005. Visions conflictuelles du développement durable dans les négociations sur le changement climatique. In Le développement durable : une perspective pour le $21^{\text {ème }}$ siècle (directeurs : Maréchal J.-P. et Quenault B.), PUR, p.p. 359-378.

Quenault B., 2006. Protocole de Kyoto et gouvernance écologique mondiale: enjeux et perspectives des engagements post-2012. Mondes en Développement, $\mathrm{n}^{\circ}$ 136, p. 29-47.

Riedacker A., 2003. Effet de serre et politiques de lutte contre le changement climatique. Mondes en Développement, $\mathrm{n}^{\circ} 121, \mathrm{p} .47-70$.

Smouts M.C. (sous la direction de), 2005. Le développement durable. Les termes du débat. Armand Colin, 289 p. Tabeaud M., 2008. Le changement climatique : une stratégie internationale d'atténuation? Dans Atlas des développements durables (dir. Veyret Y. et Arnould P.), p.56-57.

Theys J., 2001. A la recherche du développement durable : un détour par les indicateurs. Dans Le développement durable, de l'utopie au concept (ed. Jollivet), p. 269-280.

Tsayem Demaze M., 2008a. Les conventions internationales sur l'environnement : état des ratifications et des engagements des pays, soumis à L'Information Géographique (Doc IG)

Tsayem Demaze M., 2008b. Paradoxes conceptuels du développement durable et nouvelles initiatives de coopération Nord-Sud : le mécanisme pour un développement propre. Accepté pour publication dans Cybergéo

UNFCCC, 2007. Données présentées dans les inventaires nationaux de gaz à effet de serre pour la période 19902005, 43 p (http://unfccc.int/resource/docs/2007/sbi/fre/30f.pdf).

Veyret Y. (dir.), 2007. Le développement durable. SEDES, 432 p.

Veyret Y., Arnould P.(dir.), 2008. Atlas des développements durables. Un monde inégalitaire, des expériences novatrices, des outils pour l'avenir. Editions Autrement, 87 p.

Wackernagel M., Rees W., 1999. Notre empreinte écologique. Ecosociété, 207 p.

WWF, 2008. Rapport planète vivante 2008, 44 p. (http://www.wwf.fr)

WWWF., 2006. Rapport planète vivante 2006. 40 p. (http://www.wwf.fr). 